\title{
Influencia de las diferentes etapas de procesamiento sobre la microestructura de dispositivos multicapa basados en PZT
}

\author{
E. SOLERA, T. JARDIEL, M. VILLEGAS, J.F. FERNÁNDEZ \\ Departamento de Electrocerámica. Instituto de Cerámica y Vidrio, CSIC 28500 Arganda del Rey, Madrid.
}

\begin{abstract}
Se ha realizado un estudio comparativo de la influencia de los parámetros reológicos y de procesamiento sobre la microestructura y consistencia de chips multicapa de PZT obtenidos mediante un proceso de colado en cinta. Para ello se han realizado medidas de viscosidad de distintas barbotinas con diferente contenido en sólidos y su relación con el espesor y densidad en verde de las cintas obtenidas, se han identificado los puntos críticos del ciclo de quemado-sinterización y, mediante MEB se ha estudiado la microestructura en verde de las láminas coladas y la microestructura final de los chips multicapa fabricados.
\end{abstract}

Palabras clave: reología, colado en cinta, multicapa, PZT

Influence of the different processing steps on the microstructure of PZT-based multilayer

A comparative study of the influence of both rheological and processing parameters on the microstructure and reliability of multilayer ceramic chips based on PZT has been conducted. The multilayer chips were obtained by tape casting. Viscosity measurements of different slurries with various solids content have been correlated with both the thickness and the green density of the casted layers. The critical points of the thermal treatments, organics burn-out and sintering, have been identified. The green microstructure of the layers and the final microstructure of the sintered chips have been studied by SEM.

Key words: rheology, tape-casting, multilayer, PZT

\section{INTRODUCCIÓN}

Los materiales cerámicos basados en titanato circonato de plomo (PZT) $(1,2)$ dominan el campo de los materiales piezoeléctricos para el diseño de la mayoría de los dispositivos requeridos en la industria, fundamentalmente su aplicación como sensores y actuadores piezoeléctricos, tanto en conformado masivo como en dispositivos multicapa $(3,4)$.

Las estructuras piezoeléctricas multicapa se preparan mediante procesos basados en la tecnología de fabricación de Condensadores Cerámicos Multicapa $(5,6)$ en la que se emplea la técnica de colado en cinta para obtener láminas cerámicas en verde. Sin embargo, los requerimientos del material y del proceso en los multicapas piezoeléctricos son algo más críticos que en el caso de los condensadores debido a que tienen la exigencia añadida de funcionar activamente como un dispositivo mecánico. Este requerimiento añadido es la razón principal de las modificaciones en el diseño, de las cuales las principales se centran en la mejora de la distribución de tensiones y la integridad mecánica de las diferentes uniones del mismo (7).

El uso de la tecnología de colado en cinta exige la preparación de una barbotina de polvo cerámico con una composición ajustada de los distintos aditivos lo cual permite fabricar láminas de espesor y densidad en verde adecuados. También es necesario un estudio reológico preciso de dichas barbotinas para lograr una elevada fiabilidad y reproducibilidad del proceso. En este sentido, además del control del polvo cerámico de partida: tamaño y distribución de tamaño de partícula, superficie específica, estado de aglomeración y forma de las partículas, es necesario conseguir una buena dispersión del polvo en la barbotina, para lo cual se emplean aditivos orgánicos (8-10) y procesos de molienda que permiten la homogeneización de los diferentes componentes de la suspensión, así como la reducción de los aglomerados que pudieran estar presentes, además de impedir la floculación y/o agregación de las partículas.

Tras el proceso de colado en cinta, es necesaria una etapa de secado de las cintas coladas en la que la concentración de aditivos orgánicos es un parámetro fundamental para las propiedades finales de las cintas. Las etapas de apilado y corte, así como el tratamiento térmico posterior de los chips son etapas críticas en las que pueden producirse gran cantidad de defectos que finalmente harán que los chips piezoeléctricos no sean utilizables. El tratamiento térmico consta de dos etapas: la primera, a baja temperatura, donde se produce el quemado y eliminación de los aditivos orgánicos y la segunda, a temperaturas mayores donde se produce la sinterización de los chips.

El objetivo de este trabajo ha sido determinar mediante un estudio reológico y microestructural la composición más adecuada de una barbotina de polvo cerámico basado en PZT para obtener cintas coladas con propiedades óptimas que conduzcan a chips libres de defectos que puedan aplicarse con éxito como dispositivos piezoeléctricos multicapa. 


\section{PROCEDIMIENTO EXPERIMENTAL}

Se prepararon seis suspensiones de polvo cerámico con diferente contenido en sólidos a partir de un PZT tipo 5A sintetizado como se describe en (11). El polvo cerámico se suspendió en etanol y mediante agitación mecánica con el empleo de una turbina de alta velocidad de cizalla se mezcló con esterfosfato (EP) como dispersante. La mezcla de polvo cerámico y dispersante se secó en una estufa a $60^{\circ} \mathrm{C}$ y una vez tamizada a través de una malla de $100 \mu \mathrm{m}$ se mezcló con una disolución compuesta por un disolvente (mezcla azeotrópica metiletilcetona-etanol, MEK-ET 66-34), un aglomerante (polivinilbutiral, PVB) y plastificantes (polietilenglicol, PEG, y bencilbutilftalato, BBP). La mezcla se llevó a cabo en un molino de bolas de circona durante 2 horas. Tras la molienda se realizó un tratamiento de desaireación a vacío durante 2 minutos para eliminar las posibles burbujas ocluidas en el interior de la barbotina. Las diferentes composiciones de las barbotinas con distinto contenido en sólidos se indican en la Tabla I.

TABla I. COMPOSICIONES EN \% EN PESO DE LAS BARbOTINAS PARA COLADO

\begin{tabular}{|c|c|c|c|c|c|c|}
\hline \multirow{2}{*}{ Componentes } & \multicolumn{6}{|c|}{ Composición de las barbotinas } \\
\cline { 2 - 7 } & C1 & C2 & C3 & C4 & C5 & C6 \\
\hline PZT & 39.23 & 49.25 & 54.21 & 55.66 & 59.20 & 64.18 \\
\hline EP & 0.07 & 0.09 & 0.10 & 0.10 & 0.11 & 0.12 \\
\hline MEK-ET & 44.75 & 37.35 & 33.71 & 31.85 & 30.02 & 26.33 \\
\hline PVB & 13.24 & 11.07 & 9.96 & 9.42 & 8.88 & 7.79 \\
\hline PEG & 0.90 & 0.75 & 0.67 & 0.69 & 0.60 & 0.53 \\
\hline BBP & 1.79 & 1.49 & 1.35 & 1.27 & 1.20 & 1.05 \\
\hline
\end{tabular}

El colado de las diferentes barbotinas se realizó empleando una mesa de colado en cinta con cuchilla móvil y substrato fijo $(12,13)$. El substrato empleado fue Mylar®. El colado se realizó a una velocidad de $39.5 \mathrm{~Hz}$ con una apertura de cuchilla de $0.5 \mathrm{~mm}$. El secado de la cinta se hizo en atmósfera de acetona. El apilado se realizó mediante prensado en caliente a una presión de 0.4 bar y una temperatura de $74^{\circ} \mathrm{C}$. Una vez laminadas las cintas se cortaron para obtener los chips individuales que en el caso de este trabajo estaban formados por 4 láminas.

El tratamiento térmico posterior de los chips obtenidos se determinó estudiando diferentes velocidades $\left(0.1\right.$ a $\left.1^{\circ} \mathrm{C} / \mathrm{min}\right)$, tiempos (30 min-3 horas) y temperaturas $\left(450^{\circ}-600^{\circ} \mathrm{C}\right)$ de la etapa quemado de orgánicos, estableciéndose las condiciones óptimas en un calentamiento a una velocidad de $0.2^{\circ} \mathrm{C} / \mathrm{min}$ hasta $200^{\circ} \mathrm{C}$ seguido de un calentamiento a una velocidad de $0.5^{\circ} \mathrm{C} / \mathrm{min}$ hasta $500^{\circ} \mathrm{C}$. Manteniéndose a esa temperatura máxima 1 hora. El ciclo de sinterización se llevó a cabo con un ciclo adecuado para dispositivos ferroeléctricos multicapa (14), es decir, a una velocidad de calentamiento de $3^{\circ} \mathrm{C} / \mathrm{min}$ hasta una temperatura máxima comprendida entre $1000^{\circ} \mathrm{y}$ $1100^{\circ} \mathrm{C}$ durante 2 horas.

La determinación de la viscosidad de las diferentes barbotinas se realizó empleando un viscosímetro rotacional
Rotovisco RV 20 que dispone de un sistema de medición Searle basado en la utilización de cilindros coaxiales y de un estabilizador de temperatura por circulación. Las medidas se llevaron a cabo a $25^{\circ} \mathrm{C}$ hasta velocidades de cizalla de $200 \mathrm{~s}^{-1}$ con un ciclo de 2 minutos hasta alcanzar $200 \mathrm{~s}^{-1}, 1$ minuto a dicha velocidad de cizalla y 2 minutos hasta $0 \mathrm{~s}^{-1}$. Para el análisis de los espesores en verde de las cintas se usó un comparador Mitutoyo. La densidad de las cintas en verde se determinó geométricamente, al igual que la contracción de los chips tras la sinterización. El análisis microestructural de las láminas y chips en verde y de los mismos una vez sinterizados se realizó mediante Microscopía Electrónica de Barrido (MEB) empleando un Microscopio Zeiss DSM950.

\section{RESULTADOS Y DISCUSIÓN}

En el proceso de colado en cinta la reología de la suspensión cerámica empleada es un parámetro básico ya que el empleo de barbotinas de elevada viscosidad permite la retención de la forma en la lámina colada. Además, las barbotinas deben poseer comportamiento pseudoplástico, es decir, suspensiones en las que la aplicación de fuerzas de cizalla altas (como las que se producen al pasar la barbotina por debajo de la cuchilla) produzcan una disminución de la viscosidad y que una vez eliminadas esas altas fuerzas de cizalla la viscosidad aumente de nuevo. La evolución de la viscosidad y comportamiento reológico de las diferentes barbotinas estudiadas se muestra en la Figura 1 en la que puede verse la evolución de la curva de viscosidad de las suspensiones en función de la velocidad de cizalla. El contenido en sólidos de las suspensiones influye fuertemente en su viscosidad ya que ésta disminuye conforme disminuye el contenido en sólidos. Además el comportamiento pseudoplástico necesario para la retención de la forma de las cintas coladas aparece sólo en las barbotinas con mayor contenido en sólidos (C4, C5 y C6) mientras que las que tienen bajo contenido en sólidos ( $\mathrm{C} 1, \mathrm{C} 2$ y C3) presentan una curva de viscosidad que se asemeja a la de un flujo newtoniano y, por lo tanto, su comportamiento no es adecuado para el proceso de colado en cinta.

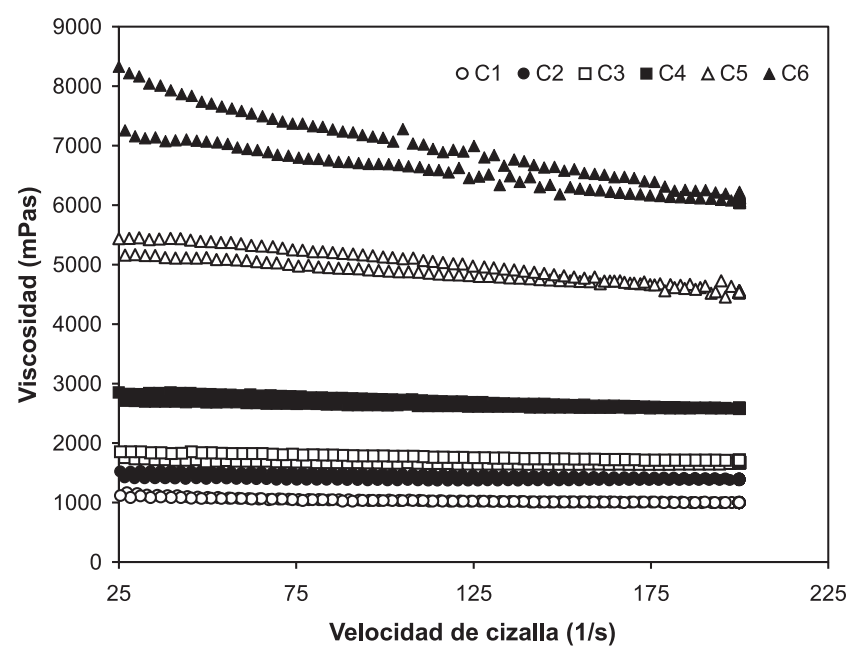

Figura 1. Curvas de viscosidad de las diferentes suspensiones de PZT-5A preparadas 
El contenido en sólidos de las barbotinas influye en tres características básicas de las cintas coladas en verde: densidad, espesor (contracción) y microestructura. En la Figura 2 puede verse como la densidad en verde aumenta con el contenido en sólidos. Este comportamiento es el esperado ya que cuanto mayor es el contenido en sólidos menor es la cantidad de aditivos orgánicos adicionados a la barbotina por lo que las partículas pueden establecer una red más cohesionada que produce el aumento de densidad. La variación del espesor de las cintas una vez secas (Figura 2) sigue un comportamiento análogo al de la densidad en verde, a pesar de que todas ellas fueron obtenidas en las mismas condiciones de velocidad y apertura de cuchilla. Por tanto, no sólo las condiciones de colado determinan el espesor de las cintas en verde sino que el contenido en sólidos es también un parámetro fundamental para la obtención de cintas de un determinado espesor ya que la cantidad de aditivos orgánicos determinan la contracción de la cinta durante la etapa de secado.

El estudio mediante MEB de las cintas en verde (Figura 3) revela que el contenido en sólidos determina también su microestructura. Así, las barbotinas con alto contenido en sólidos dan lugar a cintas con superficies muy homogéneas ya que la cantidad de disolvente que ha de evaporarse en la etapa de secado se ha minimizado, haciendo que la microestructura en verde de las mismas presente alta homogeneidad y escaso "pinchado" de la lámina (Figura 3.a). Por el contrario, en las barbotinas con menor contenido en sólidos que presentan comportamiento newtoniano se produce una segregación del exceso de disolvente y aditivos orgánicos hacia la superficie de las cintas durante la etapa de secado. La necesidad de eliminar gran cantidad de disolvente en las suspensiones de bajo contenido en sólidos produce cintas con una superficie agrietada, similar a una piel de serpiente, con la aparición de "pinchado" (Figura 3.b).

Por tanto, la mayor porosidad de las láminas de bajo contenido en sólidos hace que la densidad en verde de las mismas disminuya y esta disminución, a su vez, produce una dis-

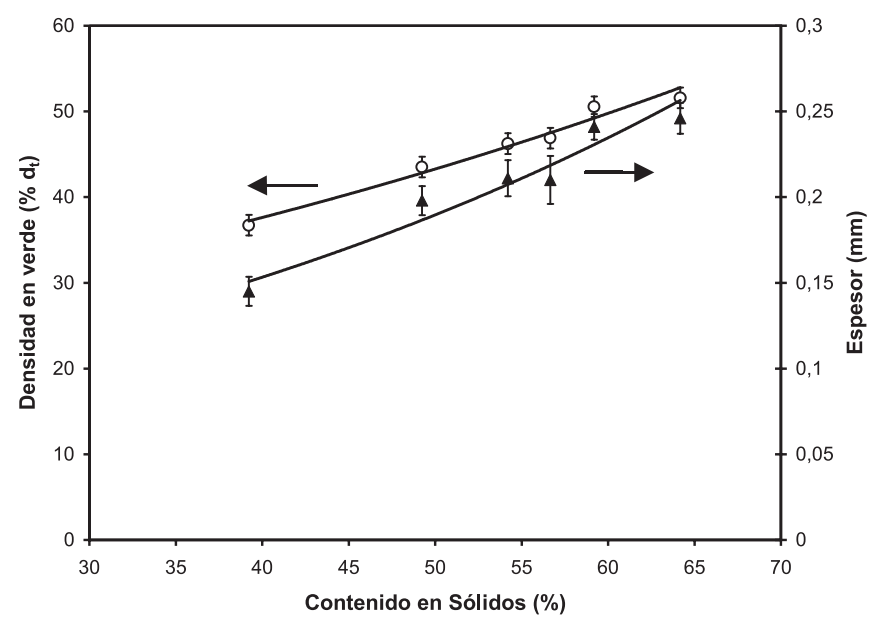

Figura 2. Variación de la densidad en verde y del espesor de las cintas coladas en función del contenido en sólidos de las barbotinas.

minución del espesor de la cinta en verde debido a las mayores contracciones producidas al eliminar una mayor cantidad de disolvente (ver Figura 2).

Como es lógico, estos datos pueden extrapolarse a los "chips" obtenidos tras la etapa de apilado y corte. Así, aquellos fabricados con cintas de bajo contenido en sólidos (C2 y C3) presentan densidades en verde y espesores menores que en los conformados a partir de láminas con alto contenido en sólidos. Además, hay que destacar que en el caso más extremo (C1) no pudieron conformarse "chips" multicapa ya que en las condiciones experimentales empleadas la heterogeneidad de la distribución de los aditivos y partículas sólidas en estas láminas no permitió un apilado correcto de las láminas en verde.
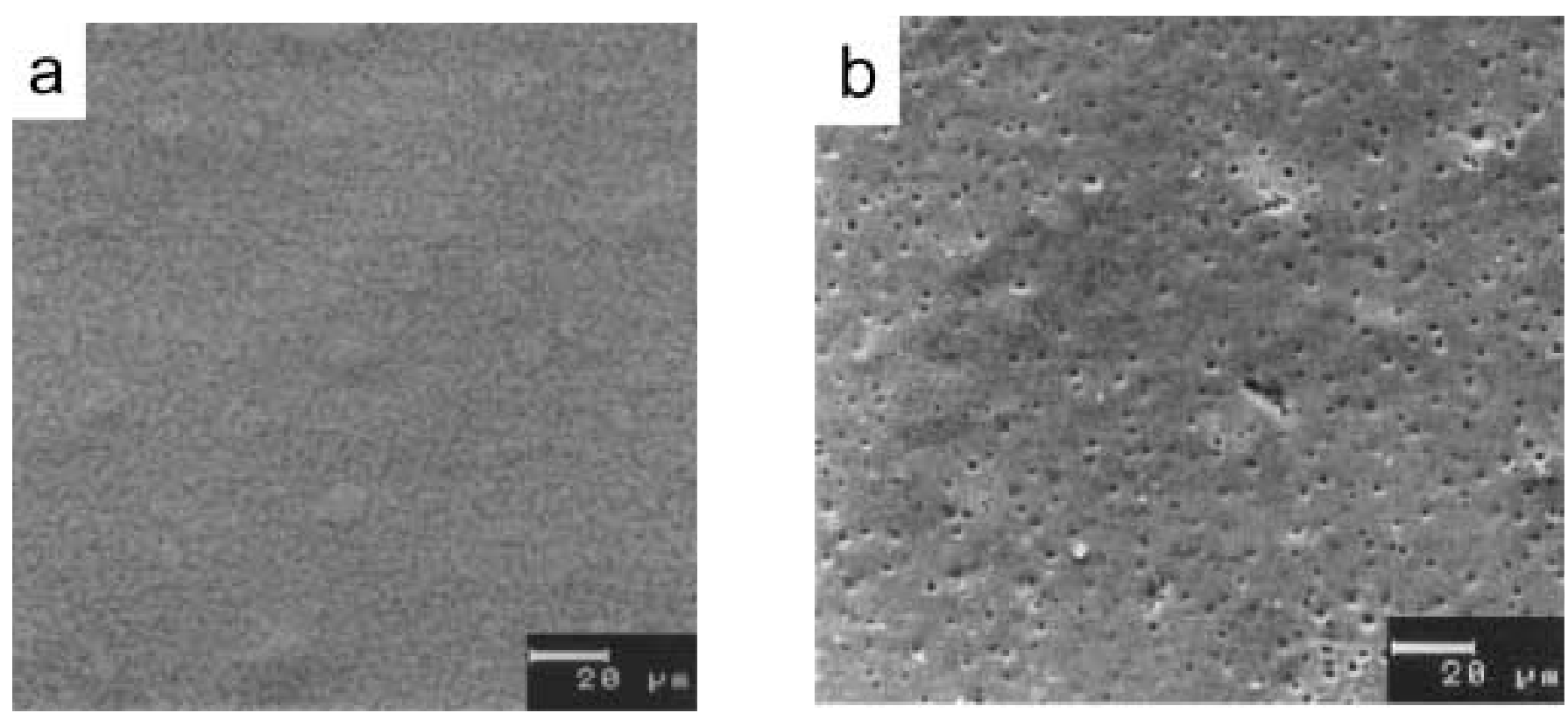

Figura 3. Micrografía de MEB de la superficie de las cintas en verde. a) cinta de alto contenido en sólidos (C1) y b) cinta de bajo contenido en sólidos (C5). 
Al igual que en las propiedades anteriores el contenido en sólidos influye directamente en las pérdidas de peso experimentadas por los chips durante el tratamiento térmico. En la Figura 4 puede verse la variación de las pérdidas de peso de los chips tras la etapa de quemado de orgánicos en función del contenido en sólidos. El proceso de eliminación de los aditivos orgánicos empleados en la formulación de la barbotina debe ser completo y realizarse de forma gradual para permitir la correcta eliminación de los gases producto de su combustión. Esta eliminación implica la obtención de "chips" libres de defectos y agrietamientos producidos por la evolución de gases, por lo que la eliminación de éstos debe ser muy lenta y, además, el contenido de carbono residual debe minimizarse ya que resulta perjudicial para las propiedades eléctricas finales del multicapa. Por tanto, las barbotinas con bajo contenido en sólidos (C2 y C3) no son adecuadas para la fabricación de los chips ya que la concentración de aditivos que debe eliminarse es muy elevada y, en las condiciones del ciclo óptimo de quemado, los gases procedentes de la combustión no se eliminan correctamente dando lugar a abombamientos superficiales de los chips y a delaminaciones de las capas que los conforman (Figura 5). Además, aunque no se ha determinado en este trabajo, el contenido de carbono residual será, lógicamente, más elevado que en el caso de los chips procedentes de barbotinas con alto contenido en sólidos.

Durante la etapa de sinterización los problemas encontrados durante la etapa de eliminación de orgánicos en las composiciones $\mathrm{C} 2$ y C3 se hacen más evidentes especialmente en el caso de la C2 donde aparecen problemas de contracción (Figura 6). Unicamente las composiciones C5 y C6 presentan una contracción aceptable durante la sinterización ya que su elevada densidad en verde hace disminuir la contracción lo que se traduce en una elevada estabilidad dimensional de los chips y, por tanto, en una disminución de la probabilidad de aparición de defectos. En las Figura 7.a y 7.b se muestra las micrografías de MEB de un chip de la composición C6 en la que puede verse su mayor densidad y homogeneidad final comparado con el caso de la composición de bajo contenido en sólidos, C3, (Figura 7.c) en donde son evidentes las delaminaciones y defectos generados por la alta contracción experimentada.

\section{CONCLUSIONES}

Se han estudiado las condiciones óptimas para realizar el colado en cinta de materiales piezoeléctricos basados en PZT$5 \mathrm{~A}$ con objeto de obtener chip multicapa sinterizados con buena reproducibilidad y microestructura homogénea y libres de defectos tras la sinterización a baja temperatura $\left(1000^{\circ} \mathrm{C} / 2 \mathrm{~h}\right)$. Los estudios reológicos de las distintas barbotinas ensayadas revelan que es necesario un elevado contenido en sólidos $(>55 \%)$ para poder obtener suspensiones con una viscosidad elevada y el comportamiento pseudoplástico necesario para llevar a cabo el colado de las cintas. Las barbotinas con alto contenido en sólidos permiten obtener cintas coladas con microestructura homogénea, en comparación con la microestructura heterogénea y con pinchado de las cintas obtenidas a partir de suspensiones con bajo contenido en sólidos $(<55 \%)$. La baja densidad en verde de las láminas de bajo contenido en sólidos y el exceso de componentes orgánicos impiden la obtención de chips multicapa tras el laminado de las cintas. Con un contenido en sólidos intermedio es posible fabricar chips, sin embargo, durante la etapa de quemado de orgánicos se producen delaminaciones que se hacen más evi-

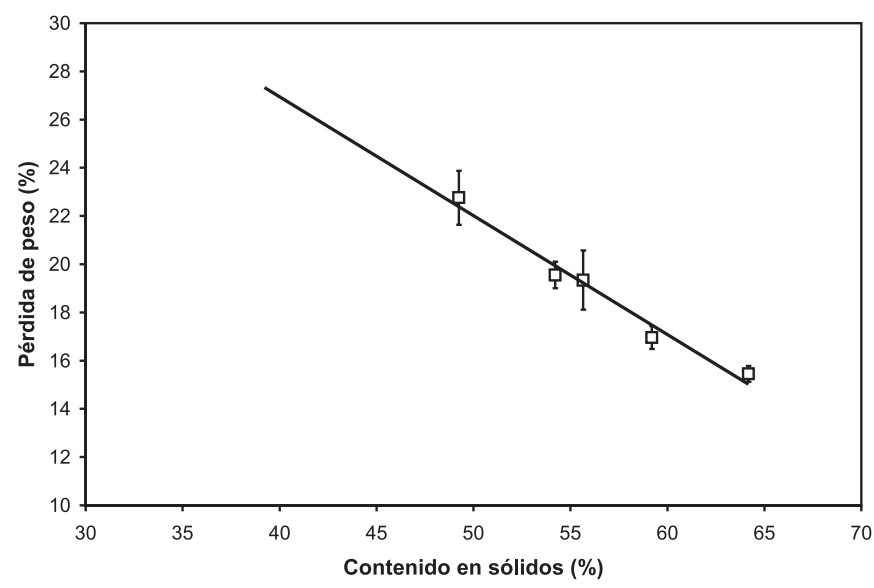

Figura 4. Pérdida de peso durante la calcinación de los "chips" (500ㄷ $/ 1 \mathrm{~h})$.

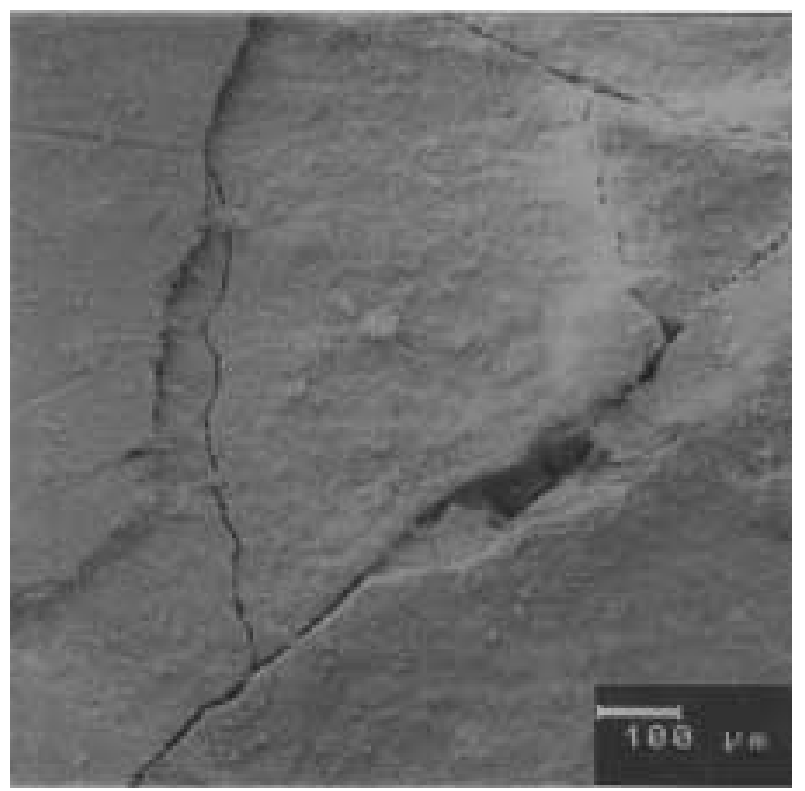

Figura 5. Micrografía de MEB del detalle de la delaminación de un "chip" (C3) después del tratamiento de calcinación

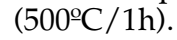

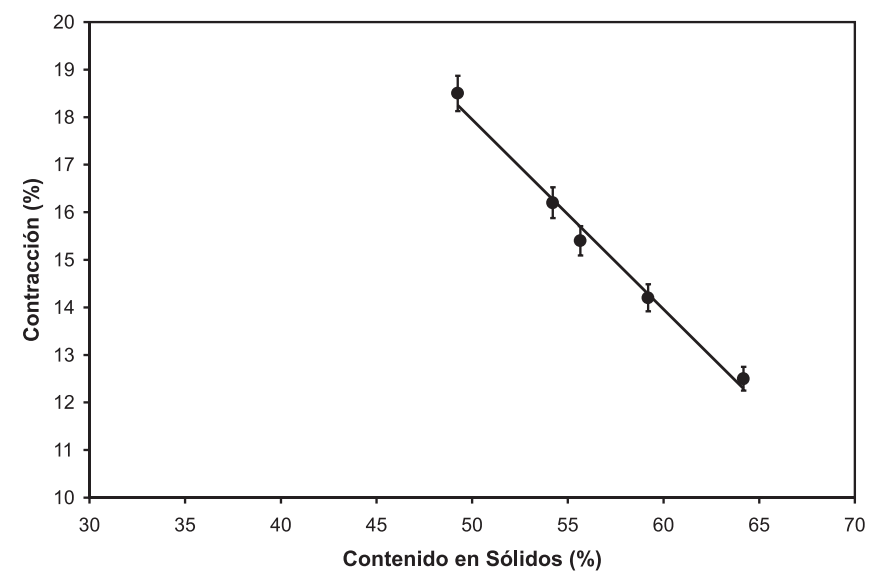

Figura 6. Contracción de los "chips" durante la sinterización ( $\left.1000^{\circ} \mathrm{C} / 2 \mathrm{~h}\right)$ en función del contenido en sólidos. 

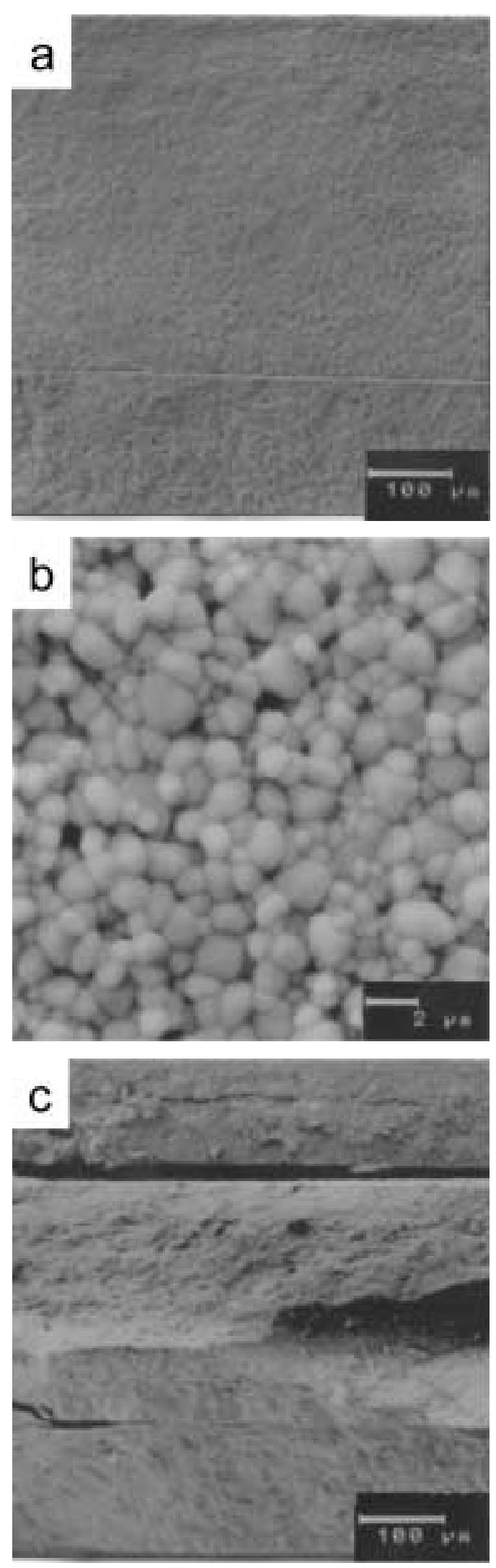

Figura 7. Micrografías de MEB de "chips" sinterizados $\left(1000^{\circ} \mathrm{C} / 2 \mathrm{~h}\right)$.

a) Fractura transversal de un "chip" C6.

b) Superficie de un "chip" C6.

c) Fractura transversal de un "chip" C3. dentes tras la sinterización y que, por tanto, hace que dichos chips no puedan emplearse en dispositivos electromecánicos. Las láminas con alto contenido en sólidos presentan muy buen comportamiento durante el laminado, el quemado de orgánicos y la sinterización, permitiendo la obtención de chips multicapa sin defectos estructurales y con una microestructura muy homogénea.

\section{AGRADECIMIENTOS}

Los autores quieren agradecer al CSIC y al Proyecto EUREKA 2309 FACTORY-PAMIS su apoyo en la realización de este trabajo.

\section{REFERENCIAS}

1. C. Wenrworth, G.W. Taylor. "Processing Parameters and Electrical Properties of Doctor-Blade Ferroelectric Ceramics". Am. Ceram. Soc. Bull., 46(12), 11861193 (1967).

2. M. Villegas, C. Moure, J.R. Jurado, P. Durán. "Efecto de la Adición de Coloides sobre la Sinterización y Propiedades Piezoeléctricas de Materiales de PZT". Bol. Soc. Esp. Ceram. Vidr. 30, 357-363 (1991)

3. S. Takahashi, T. Yano, I. Fukui, E. Sato. "Multilayer Piezoelectric Ceramic Actuator with Varying Thickness Layers". Jpn. J. Appl. Phys., 24, 206-208 (1985).

4. R. Goldberg, S. Smith. "Multilayer Piezoelectric Ceramics for TwoDimensional Array Transducers". IEEE Trans. Ultrason., Ferroelec., Freq. Contr., 41, 761-771 (1994).

5. G.R. Howatt, R.G. Breckeridge, J.M. Brownlow. "Fabrication of Ceramic Thin Sheets for Capacitors". J. Am. Ceram. Soc., 30(8), 237-242 (1947).

6. R.E. Mistler. "Tape Casting: The Basic Process for Meeting the Needs of the Electronic Industry". Ceram. Bull. 69(6), 1022-1026 (1990).

7. H. Aburatani, S. Harada, K. Uchino, A. Furuta, Y. Fuda. "Destruction Mechanism in Ceramic Multilayer Actuators". Jpn. J. Appl. Phys. 33, 30913094 (1994).

8. R. Moreno, J. Requena. "Introducción al Colaje en Cinta". Bol. Soc. Esp. Ceram. Vidr., 31(2), 99-108 (1992).

9. J.C. Williams. "Doctor Blade Process". pp. 133-178 en Teatrise on Materials Science and Technology. Vol. 9. Ceramic Fabrication Process. Ed. F.Y.Y. Wang. Academic Press, New York 1976.

10. E.P. Hyatt. "Making Thin, Flat Ceramics - A Review". Am. Ceram. Soc. Bull., 65[4], 637-368 (1986).

11. L. A. Celi, A. C. Caballero, M. Villegas, P. Durán, C. Moure, J. F. Fernández. "Efecto de las Características de los Polvos Cerámicos sobre la Densificación de Materiales Cerámicos PZT". Bol. Soc. Esp. Ceram. Vidr., 38 (5) 493-497 (1999).

12. R.B. Runk, M.J. Andrejco. "A Precision Tape Casting Machine for Fabricating Thin Ceramic Tapes". Am. Ceram. Soc. Bull., 54(2), 199-200 (1975)

13. J. Jiménez, M. Poyato, J.F. Fernández. “Sistema de Precisión para Colado en Cinta de Materiales Cerámicos Avanzados". Bol. Soc. Esp. Ceram. Vidr., 33(1), 44-52 (1994).

14. M. Villegas, J.F. Fernández, C. Moure, P. Durán. "Preparation, Microstructural Development and Dielectric Properties of $\mathrm{Pb}\left(\mathrm{Mg}_{1 / 3} \mathrm{Nb}_{2 / 3}\right) \mathrm{O}_{3}$ $\mathrm{Pb}\left(\mathrm{Ti}_{x} \mathrm{Zr}_{1-\mathrm{x}}\right) \mathrm{O}_{3}$ Multilayer Ceramic Capacitors". J. Mat. Sci., 29, 4999-5004 (1994).

Recibido: 31.05 .01

Aceptado: 10.12 .01 